3. Kapitel

\title{
Geschlecht und Gesundheit
}

\author{
Monika Sieverding
}

\section{Einleitung: Morbidität und Mortalität im Geschlechtervergleich}

Für die Vorhersage von gesundheitsrelevantem Verhalten und körperlicher Gesundheit und Krankheit ist das Geschlecht neben Alter und sozialer Schicht ein besonders relevanter Prädiktor. Männer verhalten sich deutlich gesundheitsriskanter und handeln weniger präventiv für ihre Gesundheit. Damit einhergehend gibt es große Geschlechtsunterschiede in der Anfälligkeit für Krankheiten sowie in der Lebenserwartung.

Frauen erkranken häufiger an nicht lebensbedrohlichen chronischen Krankheiten wie chronischer Sinusitis, Arthritis, Allergien, Schilddrüsenerkrankungen, Gallenblasenerkrankungen oder Migräne. Männer sind dagegen häufiger von akuten und lebensbedrohlichen Krankheiten betroffen, wie Arteriosklerose, koronarer Herzkrankheit, AIDS, Lungenkrebs oder Lungenemphysem sowie Leberzirrhose (Robert-Koch-Institut, 2000). Frauen haben in allen Industriegesellschaften eine höhere durchschnittliche Lebenserwartung und eine niedrigere Gesamtsterblichkeit als Männer. In den meisten westlichen Industrieländern liegt die Differenz zwischen fünf und acht Jahren, in Deutschland leben Frauen im Durchschnitt sechs Jahre länger als Männer (80.8 vs. 74.8 Jahre; Statistisches Bundesamt, 2002).

Unter gesundheitspsychologischen Gesichtspunkten besonders relevant ist die Übersterblichkeit der Männer im mittleren Lebensalter. In Deutschland starben im Jahr 2000 doppelt so viele Männer (108.649) wie Frauen (52.864) im Alter zwischen 25 und 65 Jahren (Statistisches Bundesamt, 2002). Männer zwischen 25 und 65 Jahren sterben häufiger als Frauen an bösartigen Neubildungen (Geschlechterverhältnis $1.4: 1$ ), insbesondere an bösartigen Neubildungen 
der Atmungsorgane (3.3:1), an koronarer (ischämischer) Herzkrankheit ( $4: 1$ ), an Leberkrankheiten $(2.5: 1)$ und auf Grund von äußeren Ursachen wie Transportmittelunfällen $(3.4: 1)$ oder vorsätzlicher Selbstbeschädigung $(3.3: 1)$.

Bei diesen Todesfällen handelt es sich zu weiten Teilen um so genannte „vermeidbare Todesfälle", definiert als Sterbefälle, die nach heutigem medizinischen Kenntnisstand in bestimmten Altersgruppen (ca. bis zu 65 Jahren) erfolgreich behandelt werden könnten bzw. die durch individuelles Verhalten oder Prävention hätten verhindert werden können. Dies trifft nicht nur für die koronare Herzkrankheit (KHK) zu, sondern gleichermaßen auf die bösartigen Erkrankungen der Atmungsorgane, die Leberkrankheiten sowie die Sterbefälle auf Grund von äußeren Ursachen. Die Übersterblichkeit der Männer im mittleren Lebensalter ist somit weniger ein „Schicksal“", sondern ein vermeid- und änderbares Phänomen.

Obwohl biologische Faktoren (hier insbesondere Geschlechtschromosomen und Geschlechtshormone) zweifellos an Geschlechtsunterschieden in der Morbidität und Mortalität beteiligt sind (s. dazu Waldron, 1983), können sie diese jedoch allein nicht erklären. Die Todesursachen, die die größten Geschlechtsunterschiede aufweisen - Transportmittel-Unfälle, Lungenkrebs, Mord, Selbstmord, Leberzirrhose und AIDS - sind eindeutig in Verhaltensweisen und kaum in der Biologie begründet. Die historische und kulturelle Variation in der Größe des Geschlechtsunterschiedes im KHK-Risiko wie in der Lebenserwartung im weltweiten Vergleich ist ein weiterer Indikator für die Grenzen des biologischen Erklärungsansatzes. So ist die höhere Lebenserwartung der Frauen in westlichen Industriestaaten historisch gesehen ein relativ neues Phänomen; beispielsweise hatten in Europa bis ins Mittelalter hinein Männer eine geringere Sterblichkeit als Frauen. Auch heute gibt es noch Länder, in denen Frauen keine längere Lebenserwartung aufweisen als Männer (z. B. Pakistan oder Nepal). Der dramatische Rückgang der (männlichen) Lebenserwartung, der innerhalb eines sehr kurzen Zeitraums in Osteuropa, besonders deutlich in Russland stattgefunden hat, lässt sich kaum durch biologische Faktoren, sondern nur durch psychosoziale Variablen (insbesondere des Lebensstils) erklären (Weidner, Kopp \& Kristenson, 2002).

In der (medizin-)soziologischen Forschung ist das Thema „Geschlecht und Gesundheit “ - zunächst mit einem Schwerpunkt auf der Frauengesundheit - schon länger aktuell. Hier wurde vor allem der Zusammenhang zwischen sozialer Lage und Gesundheit untersucht. Die Berücksichtigung von sozialen Faktoren kann beispielsweise dazu beitragen, die - vor allem in älteren Studien gefundene schlechtere subjektive Gesundheit von Frauen aufzuklären: Wenn nämlich soziodemografische Variablen wie Alter, Einkommen oder Bildung berücksichtigt werden, ist der Geschlechtsunterschied in den meisten Fällen nicht mehr 
signifikant (Macintyre, Hunt \& Sweeting, 1996). Variablen der sozialen Schicht sind jedoch weniger geeignet, die Geschlechtsunterschiede im KHK-Risiko und in der Lebenserwartung aufzuklären. Die soziale Lage von Frauen ist im Durchschnitt schlechter als die von Männern, wie sich leicht an den zentralen Merkmalen von sozialer Lage - Erwerbsbeteiligung, Einkommen und Armut - festmachen lässt (Statistisches Bundesamt, 2002).

\section{Geschlechterrollen und Gesundheit}

Die Unterscheidung von "sex" als biologischem Geschlecht und "gender" als sozial konstruiertem Geschlecht ist für die Analyse von Gesundheit und insbesondere von gesundheitsrelevantem Verhalten von zentraler Bedeutung. "Sex“ beinhaltet die biologischen Unterschiede zwischen Männern und Frauen, dagegen umfasst der Begriff "gender" alle kulturellen Vorstellungen und Erwartungen über Verhaltensweisen und Eigenschaften, die als „angemessen“ für Männer oder Frauen angesehen werden (Deaux \& LaFrance, 1998). Es gibt keine gelungene deutsche Übersetzung des Begriffes ,gender", am ehesten wird das damit umschriebene Konstrukt durch den Begriff „Geschlechterrolle" erfasst. Geschlechterrollen sind komplexe gesellschaftliche Erwartungen an das Rollenverhalten und an Rollenattribute von Männern und Frauen. Man unterscheidet grob zwei Ebenen: (a) ein System von Aufgaben und Verhaltensregeln in der Familie wie in der Gesellschaft (Rollenverhalten) und (b) normative Erwartungen an psychologische Charakteristika (Rollenattribute). Diese Erwartungen knüpfen am biologischen Geschlecht an, sind jedoch gesellschaftliche Konstruktionen, Zuschreibungen, die historischen und kulturellen Wandlungen unterworfen sind (Deaux \& LaFrance, 1998). Was haben nun Geschlechterrollen mit Gesundheit zu tun? Es besteht weit gehend Konsens darüber, dass die Geschlechtsunterschiede in physischer Gesundheit und Krankheit am stärksten durch Geschlechtsunterschiede in gesundheitsrelevantem Verhalten, insbesondere im höheren Risikoverhalten von Männern, begründet sind. Gesundheitsrelevantes Verhalten wird durch eine Vielzahl von soziokulturellen Faktoren beeinflusst; gesellschaftlichen Gender-Konstrukten (oder Geschlechterrollen) wird in diesem Zusammenhang eine Schlüsselrolle zugeschrieben. Gesellschaftliche Konstruktionen, die Männlichkeit nach wie vor über das Streben nach Macht, Status und Privilegien sowie über persönliche Attribute wie Unabhängigkeit, Stärke oder „Toughness“ definieren, fördern Risikoverhalten bei Männern und unterminieren präventives Gesundheitsverhalten (Courtenay, 2000). Dagegen "verlangt" die traditionelle weibliche Rolle, welche an Frauen auch heute noch die Hauptverantwortung für das Wohlergehen der Kinder delegiert, dass Frauen stärker auf ihre eigene Gesundheit sowie die ihrer Familie achten und den Konsum von Alkohol und Drogen meiden (Waldron, 1988). Gesellschaftliche Geschlechterrollen richten sich ganz allgemein an die „Träger" der 
Positionen männliches oder weibliches Geschlecht in einem bestimmten soziokulturellen Kontext. Aus psychologischer Perspektive stellt sich die Frage, wie Geschlechterrollen das individuelle Erleben und Verhalten einer Person beeinflussen können. In diesem Zusammenhang interessiert besonders: Wie „wirken“ Geschlechterrollen auf die Gesundheit?

\section{Ein psychologisches Modell: Wie „wirken" Geschlechterrollen auf die Gesundheit?}

In Abbildung 1 ist ein einfaches heuristisches Modell zum Zusammenhang zwischen Geschlechterrollen und Gesundheit dargestellt. Die Grundannahme dieses Modells besteht darin, dass gesellschaftliche Gender-Konstruktionen (Geschlechterrollen) vermittelt über psychologische Variablen und gesundheitsrelevantes Verhalten die physische (wie auch psychische) Gesundheit beeinflussen können. Unter gesellschaftlichen Gender-Konstrukten wird hier die Aufteilung von gesellschaftlichen Positionen und Rollen in Abhängigkeit vom Geschlecht verstanden sowie Geschlechterstereotype, d. h. Vorstellungen über angemessene und erwünschte Verhaltensweisen und persönliche Charakteristika von Männern und Frauen. Solche Gender-Konstrukte beeinflussen das Individuum einerseits direkt, andererseits über das Verhalten der sozialen Umgebung (Deaux \& LaFrance, 1998).

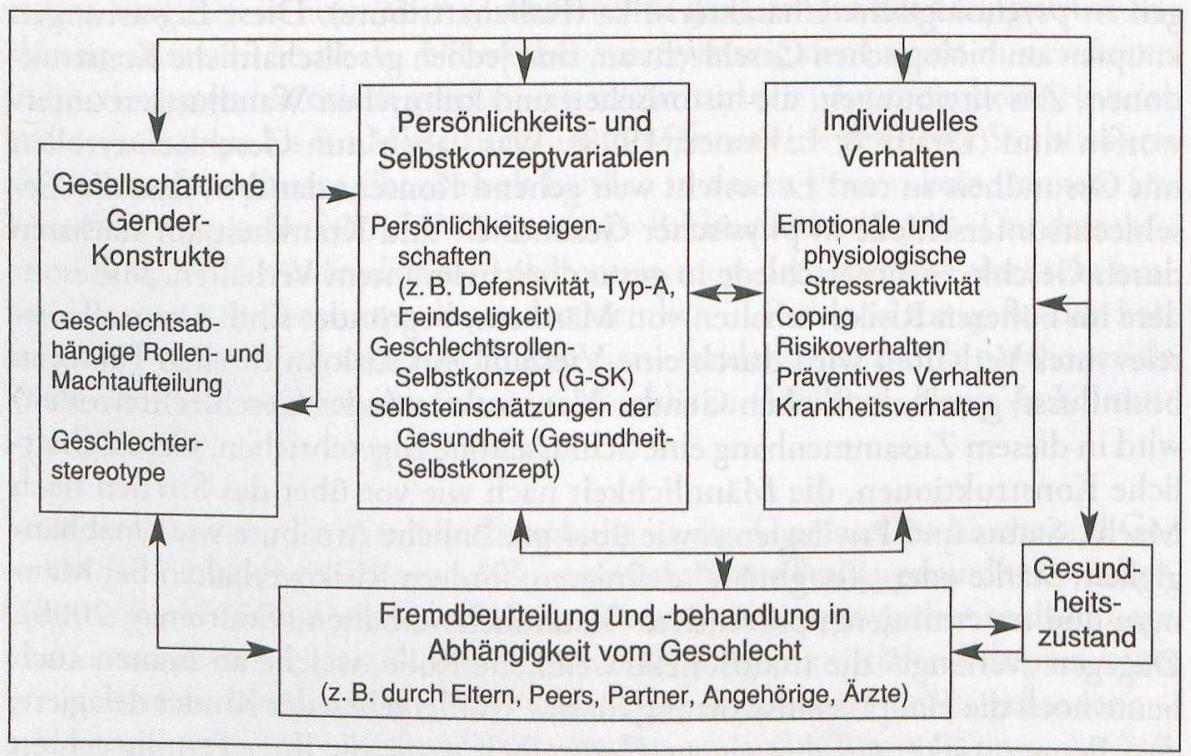

Abbildung 1:

Geschlechterrollen und Gesundheit: Ein psychologisches Modell 
Geschlechterrollen und -stereotype können sich direkt auf gesundheitsrelevantes Verhalten, z. B. gesundheitliches Risikoverhalten wie Rauchen oder Alkoholkonsum, auswirken, und zwar insbesondere dann, wenn es klare geschlechtsabhängige "Gebote" oder „Verbote" gibt. Beispiele sind der Gruppendruck in Richtung starken Trinkens in so genannten „Männerbünden“ oder die gesellschaftliche Ächtung des Rauchens bei Frauen bis in die Mitte des letzten Jahrhunderts (s. Waldron, 1988). Indirekt wirken Geschlechterrollen und -stereotype, indem sie durch Prozesse der Sozialisation, Erziehung und sozialen Interaktion die Entwicklung von individuellen Merkmalen wie Persönlichkeitsmerkmalen, Geschlechtsrollen-Selbstkonzept und gesundheitsrelevanten Selbsteinschätzungen (Gesundheits-Selbstkonzept) bei Männern und Frauen beeinflussen. Diese individuellen Merkmale wiederum können sich über verschiedene vermittelnde Pfade auf die Gesundheit auswirken. Ein Pfad führt über gesundheitsrelevantes Verhalten (Risikoverhalten, Coping, gesundheitsförderndes Verhalten), ein anderer über emotionale und physiologische Stressreaktivität (Kohlmann, 1997). So ist die bewusst schwer manipulierbare kardiovaskuläre Stressreaktivität mit Persönlichkeitsmerkmalen wie Defensivität (Kohlmann, 1997) oder Feindseligkeit (Vögele, Jarvis \& Cheeseman, 1997) assoziiert. In dem hier vorgestellten Modell wird angenommen, dass das gesundheitsrelevante Verhalten einer Person auch vom Verhalten (wichtiger) Interaktionspartner abhängt (z. B. Peers, Partner, behandelnde Ärzte bzw. Ärztinnen).

Auf die in dem Modell angenommenen Wechselwirkungen soll hier nicht im Detail eingegangen werden. Es ist evident, dass das Verhalten von Männern und Frauen und ihrer sozialen Umgebung sich gegenseitig beeinflussen und insbesondere auch auf gesellschaftliche Gender-Konstrukte zurückwirken, indem diese bestärkt oder modifiziert werden (s. Deaux \& LaFrance, 1998). Auch kann davon ausgegangen werden, dass der Gesundheitszustand (z. B. eine chronische Krankheit) Rückwirkungen auf die Persönlichkeit und das Selbstkonzept sowie das gesundheitsrelevante Verhalten eines Individuums hat.

Das vorgestellte Modell ist als Anregung für geschlechtssensitive Gesundheitsforschung gedacht. In den folgenden Abschnitten möchte ich exemplarisch auf einige Zusammenhänge, die in dem Modell postuliert werden, eingehen.

\subsection{Selbsteinschätzung von Gesundheit und körperlichen Beschwerden}

Gesundheits-Surveys, Studien zu Selbstberichten über körperliche Symptome sowie Befragungen von Ärzten erbringen in der Regel einen konsistenten Geschlechtsunterschied: Frauen berichten über mehr körperliche Beschwerden als Männer (Brähler, Schumacher \& Felder, 1999; Gijsbers van Wijk \& Kolk, 1997). 
Dieses Mehr an subjektiver Morbidität bei Frauen war einer der Gründe, die dazu geführt haben, dass Frauen bis vor kurzem als das „kränkere Geschlecht" angesehen wurden. Wie sind nun diese Geschlechtsunterschiede zu interpretieren? $\mathrm{Zu}$ nächst einmal sind drei wichtige Einschränkungen zu beachten. In vielen Studien wurde der Einfluss von Beschwerden, die mit der Reproduktionsfähigkeit der Frau zusammenhängen, auf das Gesamtmaß der Beschwerden nicht oder nicht ausreichend kontrolliert. Ein weiterer Kritikpunkt bezieht sich auf die Konstruktion von Beschwerdebögen. So wird kritisiert, dass viele Beschwerdebögen einen femininen Bias aufweisen, weil typisch weibliche Beschwerden überrepräsentiert sind, während typisch männliche Beschwerden zu wenig Beachtung finden. Eine dritte Einschränkung betrifft die Reliabilität der Selbsteinschätzungen. So sind die Geschlechtsunterschiede unter anderem von dem Befragungszeitraum abhängig. Je länger der erfragte Zeitraum ist, für den körperliche Beschwerden erinnert werden sollen, desto größer ist der Geschlechtsunterschied (Pennebaker, 1982). Ähnliche Ergebnisse findet man übrigens regelmäßig bei Selbstangaben zu körperlichen Krankheiten. Besonders relevant sind jedoch die Veränderungen in den Geschlechtsunterschieden im Lauf der Zeit. Die Tatsache, dass sich der mit der Gießener Beschwerdeskala erfasste Geschlechtsunterschied im Beschwerdedruck in Deutschland von 1975 bis 1994 gravierend vermindert hat (Brähler et al., 1999), verweist auf die Bedeutsamkeit sozialer und psychologischer Faktoren für Selbsteinschätzung und Selbstbericht von körperlichen Beschwerden. Die zunehmende Angleichung der Selbstberichte über körperliche Beschwerden hängt vermutlich mit Veränderungen in den Geschlechterrollen, insbesondere einer zunehmenden Erwerbstätigkeit bei den Frauen, zusammen.

Zur Erklärung des letztgenannten Phänomens eignet sich das Modell von Gijsbers van Wijk und Kolk (1997), welches sich eng an die Theorie der Symptomwahrnehmung von Pennebaker (1982) anlehnt. Die Symptomwahrnehmung wird demnach durch physiologische, psychologische und Umgebungsfaktoren beeinflusst. Die Symptomwahrnehmung ist eine Funktion von internen und externen Stimuli, wobei die Informationsaufnahme zwischen internalen und externalen potenziellen Informationsquellen oszilliert. Je stärker externe Stimuli auf ein Individuum einwirken, desto eher kann die Aufmerksamkeit von inneren Zuständen abgelenkt werden. Umgekehrt kann ein Fehlen von externen Stimuli die Aufmerksamkeit auf interne Stimuli verstärken. Das berufliche Engagement einer Person beispielsweise kann als ein so starker externer Stimulus wirken, dass körperliche Symptome weniger oder erst später wahrgenommen werden. Die Vorhersagen aus diesem Modell würden erklären, warum mit zunehmender Erwerbstätigkeit von Frauen die Differenzen im Beschwerdedruck zwischen Männern und Frauen abgenommen haben. Umgekehrt würde aus diesem Modell vorhergesagt werden, dass bei Männern die Wahrnehmung von körperlichen Beschwerden zunehmen sollte, wenn sie ihr berufliches Engagement zurückschrauben oder wenn sie arbeitslos werden. 
Es gibt noch einen zweiten Aspekt der Geschlechterrolle, der die Selbstwahrnehmung und den Selbstbericht von körperlichen Beschwerden über das Selbstkonzept beeinflusst. Sieverding (2002) überprüfte die Hypothese, dass ein Mann umso weniger körperliche Beschwerden berichtet, je mehr sein Selbstkonzept einem traditionellen („Macho“-)Männlichkeitsideal entspricht. Dazu wurde die Ähnlichkeit des Selbstkonzeptes mit dem Prototypen des Marlboro-Mannes aus der Kinowerbung, welcher in einer Vorstudie ermittelt worden war, berechnet. In einer Studie mit 450 Studierenden aus Ost- und Westdeutschland konnte die Hypothese unterstützt werden. Männer, die dem Marlboro-Mann in ihrem Selbstkonzept ähnelten, gaben deutlich weniger Beschwerden an im Vergleich zu Marlboro-Mann-unähnlichen Männern. Die Männer der letztgenannten Gruppe äußerten im Durchschnitt genauso viele Beschwerden wie die Frauen. Dieses Ergebnis verdeutlicht, dass dem Geschlechtsrollen-Selbstkonzept eine ungleich wichtigere Rolle zur Vorhersage (der Selbstangabe) von körperlichen Beschwerden zukommt als dem biologischen Geschlecht.

\subsection{Fremdeinschätzung von körperlichen Symptomen und Unterschiede in der Behandlung}

Geschlechterrollen und -stereotype wirken nicht nur auf Selbstkonzepte und Selbstbeurteilungen, sondern auch auf die Beurteilung anderer Personen. So wird vermutet, dass männliche und weibliche Patienten von Ärzten und Ärztinnen unterschiedlich wahrgenommen und behandelt werden. Der Nachweis geschlechtsabhängiger Fremdbeurteilungsbiases bei der Diagnose und Therapie von Beschwerden und Krankheiten ist jedoch sehr schwierig, da andere Einflussvariablen kaum kontrolliert werden können. Dennoch gibt es einige wenige Studien, in denen systematische Unterschiede in der Diagnose und Therapie von Patienten in Abhängigkeit von deren Geschlecht nachgewiesen wurden. Aus dem deutschsprachigen Raum liegt (meines Wissens) bisher nur eine einzige Studie vor, in der dezidiert überprüft wurde, ob Patienten in Abhängigkeit von ihrem Geschlecht unterschiedlich behandelt werden (Conen \& Kuster, 1988). Zu diesem Zweck wurden fortlaufend alle Patienten registriert, die in einem Dreimonatszeitraum (im Jahr 1982) die Medizinische Universitäts-Poliklinik in Basel mit dem Symptom Bauchschmerzen aufsuchten. Als Parameter für die Verhaltensbeurteilung der Ärzte diente der Zeitaufwand für die Erstkonsultation (von einer Krankenschwester gestoppt), die Zahl der Nachkontrollen, der betriebene diagnostische Aufwand und die Art und Weise der Therapie. Die behandelnden Ärzte, 13 männliche Assistenzärzte, waren über die Studie nicht informiert. Es zeigte sich eine Reihe von Unterschieden in der Behandlung von männlichen und weiblichen Patienten (jeweils $\mathrm{n}=25$, Durchschnittsalter 37 Jahre). Die Ärzte nahmen sich deutlich mehr Zeit für die Erstanamnese der Frauen ( 59 vs. 45 Minuten). Bei Männern kamen dagegen mehr 
invasive diagnostische Verfahren zum Einsatz; so wurden bei 11 Männern, aber nur bei 3 Frauen Endoskopien durchgeführt. Unterschiede gab es auch in der Therapie. Frauen wurden deutlich häufiger unspezifische Medikamente in Form von Quellmitteln oder Analgetika verschrieben, bei Männern dagegen erfolgte häufiger der Einsatz einer spezifischen Therapie. Bei männlichen Patienten wurde zunächst eher ein organisches Leiden vermutet, während bei weiblichen Patienten in der Regel die Diagnose "Colon irritabile“ (eine funktionelle Störung ohne morphologisches oder biochemisches Korrelat) bereits bei der ersten Konsultation gestellt wurde. Die Behandlungsunterschiede waren weder durch die korrigierten Einzeldiagnosen noch durch den Krankheitsverlauf zu rechtfertigen. Die Autoren interpretieren ihre Befunde als Indikator für einen Fremdbeurteilungsbias in Abhängigkeit vom Geschlecht des Patienten, wobei sie davon ausgehen, dass eine (weitere) Ursache für die Behandlungsunterschiede auch in unterschiedlichem Krankheitsverhalten von männlichen und weiblichen Patienten $\mathrm{zu}$ vermuten ist.

In einer prospektiven Beobachtungsstudie aus den USA (die auf den Daten von 1.500 Patienten und 350 Ärzten basiert) konnte nachgewiesen werden, dass Ärzte weiblichen Patienten mehr als dreimal so häufig eine Einschränkung ihrer alltäglichen Aktivitäten empfahlen im Vergleich zu männlichen Patienten mit äquivalenten Beschwerdemerkmalen (Safran, Rogers, Tarlow, McHorney \& Ware, 1997). Die Forschergruppe konnte weiter zeigen, dass dieser Unterschied in den therapeutischen Empfehlungen nicht auf Differenzen in der Gesundheit der Patienten und auch nicht auf deren Rollenverpflichtungen (wie Erwerbstätigkeit) zurückzuführen war. Vielmehr waren die unterschiedlichen Therapieempfehlungen das Resultat von Geschlechtsunterschieden im Krankheitsverhalten der Patienten sowie eines Geschlechterbias der behandelnden Ärzte.

Auch wenn die bisherige Forschungslage zu Fremdbeurteilungsbiases in Abhängigkeit vom Geschlecht der Patienten alles andere als befriedigend ist, kann man vermuten, dass hier komplizierte Wechselwirkungsprozesse vorliegen. Ausgehend von einer unterschiedlichen Selbstwahrnehmung und -attribution von Beschwerden sind Geschlechtsunterschiede in der Interaktion mit behandelnden Ärzten anzunehmen. Möglicherweise bieten Frauen von sich aus eher subjektive Krankheitstheorien an, in denen psychische Faktoren eine Rolle spielen. (Diese Hypothese bedarf jedoch dringend einer Überprüfung!) Umgekehrt beurteilen Ärzte und Ärztinnen die Beschwerden ihrer Patienten vor dem Hintergrund von Stereotypen über die Geschlechter, über den typischen Patienten bzw. die typische Patientin wie über bestimmte Krankheitsbilder. In einer qualitativen Studie bei Ärzten wurden Stereotype von männlichen und weiblichen Patienten erfragt. Dabei stellte sich heraus, dass Krankheitsverhalten, wie es eher von weiblichen Patienten erwartet wird: „more frequent attending ... more trivial problems ... more trivial medical speaking", von Ärzten bei männlichen Patienten als 
„unmännlich" wahrgenommen wird (Seymour-Smith, Wetherell \& Phoenix, 2002). Solche ärztlichen Erwartungen über ,angemessenes "Verhalten von $\mathrm{Pa}$ tienten und Patientinnen können wiederum das Patientenverhalten beeinflussen.

\subsection{Stressbewältigungsstil}

Geschlechtsunterschiede im Umgang mit Stress werden als eine mögliche Ursache von Geschlechtsunterschieden in physischer Gesundheit diskutiert (Weidner \& Collins, 1993). Meist werden zwei basale Formen der Stressbewältigung unterschieden, nämlich die defensive oder vermeidende Stressbewältigung und die vigilante Stressbewältigung. Diese beiden Coping-Stile ähneln den bekannten älteren Konstrukten der „Represser" und „Sensitizer" sowie neueren Konzeptionen des „Monitoring“ versus „Blunting“. Ein defensiver Stressbewältigungsstil ist durch eine Unterdrückung oder Verleugnung negativer Emotionen (insbesondere von Angst) in belastenden Situationen gekennzeichnet bei gleichzeitiger Präsentation eines emotional robusten oder „stoischen“ Selbstkonzeptes. Ein vigilanter Stressbewältigungsstil dagegen wird durch die Hinwendung der Aufmerksamkeit auf die stressbezogenen Reize und die eigenen Stressreaktionen charakterisiert (s. dazu Kohlmann, 1997). Bei Frauen wurden bisher meist höhere Ausprägungen in vigilanten Strategien der Stressbewältigung (z. B. Informationssuche, Antizipation negativer Ereignisse) festgestellt, dagegen erreichen Männer im Durchschnitt höhere Werte bei den vermeidenden Strategien (z. B. Verleugnung oder Selbstaufwertung). Beim Umgang mit kurzfristigen Belastungen scheint ein defensiver Bewältigungsstil häufig durchaus effektiv zu sein, z. B. bei der Bewältigung eines medizinischen Eingriffs oder einer Prüfung. Handelt es sich jedoch um längerfristige Belastungen (z. B. Arbeitslosigkeit oder berufliche Überforderung, chronische Krankheit), werden eher negative Konsequenzen für die Gesundheit erwartet (Kohlmann, 1997; Weidner \& Collins, 1993). So wird die Tatsache, dass Männer stärker gesundheitlich unter einer Verwitwung leiden als Frauen, auf unterschiedliche Strategien im Umgang mit der Verlusterfahrung zurückgeführt (s. Stroebe $\&$ Stroebe, in diesem Band).

Ein defensiver Bewältigungsstil könnte über die beiden Pfade Stressreaktivität und gesundheitsrelevantes Verhalten die Gesundheit beeinträchtigen. Die bei Vermeidern in einer Reihe von Studien (s. dazu Überblick bei Kohlmann, 1997) festgestellte erhöhte systolische Blutdruckreaktivität auf psychische Belastung gilt als ein Risikofaktor für die Entwicklung von Bluthochdruck und möglicherweise von koronarer Herzkrankheit. In einer weiteren Studie überprüften Kohlmann und Mitarbeiter die Hypothese, dass auch Geschlechtsunterschiede in gesundheitsrelevantem Verhalten auf Geschlechtsunterschiede in defensivem Stressbewältigungsstil zurückgeführt werden können (Kohlmann, Weidner, Dotzauer \& Burns, 1997). Sie fanden in ihrer Erhebung an Collegestudierenden, dass 
Frauen höhere (d. h. günstigere) Werte hatten auf den Skalen „self-care“, „drug avoidance" und "vehicle safety", nicht jedoch auf den Skalen "physical exercise" und ,healthy nutrition". Frauen zeigten außerdem niedrigere kognitive Vermeidung als Männer. Tatsächlich wurden die gefundenen Geschlechtsunterschiede in den Verhaltensweisen „vehicle safety" und „drug avoidance“ durch die Männer mit hohen Werten in kognitiver Vermeidung verursacht. Die Autoren vermuten, dass gesundheitsrelevante Verhaltensweisen unterschiedlich stark für „Self-Enhancement"-Prozesse instrumentalisiert werden können: Riskantes Autofahren („reckless driving“) und exzessiver Alkoholkonsum könnte von Männern mit einem hohen defensiven Stressbewältigungsstil als besonders selbstaufwertend wahrgenommen werden.

\subsection{Gesundheitsrelevantes Verhalten}

Es besteht weit gehend Konsens darüber, dass Geschlechtsunterschiede in gesundheitsrelevantem Verhalten für einen Großteil der Unterschiede in Morbidität und Mortalität verantwortlich sind. Gesundheitsrelevantes Verhalten wird definiert als ein Verhalten, von dem angenommen wird, dass es einen Effekt auf die Gesundheit ausübt (Waldron, 1988). Traditionell werden folgende Verhaltensweisen dazu gezählt: Ernährung, Konsum von Tabak, Alkohol und Drogen, Bewegung bzw. sportliche Aktivität sowie die Inanspruchnahme medizinischer Hilfe und präventiver Gesundheitsangebote. Geschlechterrollen wurden als wichtigste Ursache für die Geschlechtsunterschiede in gesundheitsrelevantem Verhalten identifiziert. Waldron (1988) demonstriert in einem fundierten Überblick, wie die Geschlechtsunterschiede in gesundheitsrelevantem Verhalten in Abhängigkeit von der jeweiligen Verhaltensweise und vom kulturellen Kontext variieren. Sie kann zeigen, dass die Geschlechtsunterschiede im Risikoverhalten weniger durch gesundheitsbezogene Motive (wie z. B. Sorge um die Gesundheit) oder durch biologische Faktoren (wie z. B. unterschiedliche Toleranz von Nikotin und Alkohol) beeinflusst werden, sondern vielmehr durch die Kompatibilität bzw. Inkompatibilität eines bestimmten Verhaltens mit der männlichen oder weiblichen Geschlechterrolle. Während viele Risikoverhaltensweisen - wie z. B. exzessiver Alkoholkonsum - bei Männern geduldet oder sogar gefördert werden, gelten sie bei Frauen als sozial unerwünscht.

In westlichen (wie auch in den meisten nichtwestlichen) Ländern engagieren Männer sich stärker in riskantem Verhalten: Sie benutzen häufiger Waffen, fahren schneller und riskanter Auto, üben riskantere Sportarten in ihrer Freizeit aus, konsumieren mehr illegale Drogen und arbeiten an gefährlicheren Arbeitsplätzen. In den meisten Ländern trinken und rauchen Männer auch mehr (s. Überblick bei Waldron, 1988, 1997). Die höhere Prävalenz solcher riskanter Verhaltensweisen gilt als eine der Hauptursachen für die kürzere Lebenserwartung 
der Männer im Vergleich zu Frauen (Weidner et al., 2002). Im Zusammenhang mit dem erhöhten KHK-Risiko und der geringeren Lebenserwartung von Männern wird dem Zigarettenrauchen und (übermäßigem) Alkoholkonsum eine besondere Bedeutung beigemessen. Deshalb soll auf diese beiden Risikoverhaltensweisen etwas ausführlicher eingegangen werden.

Zigarettenrauchen ist mit verschiedenen Facetten der traditionellen männlichen Rolle assoziiert: Raucher sind unabhängig, überlegen, unkonventionell und "Cool“, ein Image, das von Filmhelden der 50er Jahre (Humphrey Bogart) aufgebaut wurde und von Werbehelden bis heute (Gauloise-Typ, Marlboro-Mann) aufrechterhalten wird. Während noch bis Mitte des 20. Jahrhunderts in den meisten Ländern das (öffentliche) Rauchen für Frauen als „unweiblich“ sanktioniert war und dementsprechend wenig Frauen rauchten (Waldron, 1988), nahmen in den letzten Jahrzehnten die Geschlechtsunterschiede im Zigarettenrauchen in allen Altersgruppen substanziell ab. So betrug in den USA Anfang der 90er Jahre das Geschlechterverhältnis (Männer : Frauen) nur noch 1.1 bis 1.3 in verschiedenen Altersgruppen und zwischen 1.0 und 1.1 für Teenager (Waldron, 1997). Der Bundes-Gesundheitssurvey von 1998 ermittelte bei den Männern einen Raucheranteil von $37 \%$ und bei den Frauen von $28 \%$ (RobertKoch-Institut, 2000). Sehr viel geringer sind jedoch die Unterschiede in jüngeren Altersgruppen: $49 \%$ der männlichen und $44 \%$ der weiblichen 18- bis 24Jährigen rauchen. Die Zunahme des Rauchens in westlichen Industrienationen bei (jungen) Frauen wird darauf zurückgeführt, dass (a) das Zigarettenrauchen heute bei Frauen einhergehend mit einer gleichberechtigteren Position in der Gesellschaft nicht mehr als „unweiblich" sanktioniert wird (Waldron, 1988, 1997) und (b), dass sich junge Frauen in ihren idealen Selbstkonzepten heute an sehr ähnlichen Werten orientieren wie junge Männer. Auch junge Frauen wollen "tough", „cool“ oder unkonventionell sein, alles Attribute, die dem Image des Rauchens anhaften.

Etwas anders stellt sich die Situation beim Alkoholkonsum dar. Bereits in einem älteren Überblicksartikel bezeichneten Lemle und Mishkind (1989) das übermäßige Trinken (von Alkohol) als ein typisch männliches Problem, eine Sichtweise, die nach wie vor nichts von ihrer Aktualität verloren hat (s. z. B. Courtenay, 2000). Praktisch in jeder Facette von Alkoholkonsum zeigen sich signifikante Geschlechtsunterschiede: Männer konsumieren 30 bis $100 \%$ mehr Alkohol als Frauen, wenn sie bei einer bestimmten Gelegenheit trinken. Obwohl Männer weniger als die Hälfte der Bevölkerung ausmachen, konsumieren sie $75 \%$ des Alkohols. Männer trinken im Vergleich zu Frauen doppelt so häufig und sind doppelt so häufig betrunken. Die Prävalenz von Problemtrinken und Alkoholismus ist bei Männern 3- bis 4-mal höher als bei Frauen (Lemle \& Mishkind, 1989). In Deutschland finden sich ähnliche Verhältnisse, wie aus dem Sucht- und Drogenbericht des Bundesministeriums für Gesundheit her- 
vorgeht (Bundesministerium für Gesundheit, 2001). Unter männlichen Jugendlichen ist regelmäßiger Alkoholkonsum weiter verbreitet (38\%) als unter weiblichen Jugendlichen (20\%). Und auch erwachsene Männer trinken deutlich mehr Alkohol als Frauen. So gab im letzten Bundes-Gesundheitssurvey von 1998 die Mehrheit (60\%) der Frauen an, weniger als $1 / 4$ Liter Bier zu trinken, wenn sie Bier trinken, dagegen lag bei der Mehrheit der Männer die konsumierte Menge deutlich darüber (nur $4 \%$ bis $1 / 4 \mathrm{l}$, dagegen $23 \% 1 / 4$ bis $1 / 21,34 \% 1 / 2$ bis 11 und $21 \%$ mehr als 11 ). Entsprechende Ergebnisse zeigten sich in der Höhe des Weinkonsums (Robert-Koch-Institut, 2000).

Es ist wahrscheinlich, dass biologische Faktoren zum geringeren Alkoholkonsum von Frauen beitragen. Frauen haben eine niedrigere Alkoholtoleranz als Männer, was mit Unterschieden in Körpergröße und -gewicht sowie im Stoffwechsel zusammenhängt. Auch reproduktive Aufgaben während Schwangerschaft und Stillzeiten sowie die Hauptverantwortung für die Sorge der Kinder bedingen bei Frauen Einschränkungen des Alkoholkonsums (Waldron, 1997). Es wird jedoch vermutet, dass auch der Alkoholkonsum durch Geschlechterrollen beeinflusst wird. Die Konvergenzhypothese beispielsweise besagt, dass sich der Alkoholkonsum von Männern und Frauen entsprechend der Angleichung der Geschlechterrollen einander annähert. In der Werbung für alkoholische Getränke lässt sich diese Entwicklung bereits seit längerem beobachten. Aus dem Slogan „Beck’s Bier löscht Männer-Durst“ wurde dementsprechend „Beck’s Bier löscht Kenner-Durst“. Die Daten aus wiederholten Surveys in verschiedenen europäischen Ländern unterstützen die Konvergenzthese - zumindest für den relativ kurzen Zeitraum 80er bis Anfang der 90er Jahre - eher nicht. Eine Ausnahme war lediglich Finnland, wo im Zeitraum von 1984 bis 1992 bei finnischen Frauen eine signifikante Zunahme des Alkoholkonsums festgestellt wurde (Bloomfiel, Gmel, Neve \& Mustonen, 2001). Dass gerade in Finnland die Konvergenzhypothese bestätigt wurde, wird von den Autoren auf die gleichberechtigte Position von Frauen in Finnland sowie auf unterschiedliche Trinkkulturen zurückgeführt.

Auch in der Bundesrepublik Deutschland hat der Alkoholkonsum in den letzten Jahren bei Frauen zugenommen. So stieg in einem relativ kurzen Zeitraum von 1997 bis 2000 der Anteil von Frauen mit einem riskanten Alkoholkonsum (d. h. mehr als $20 \mathrm{~g}$ reiner Alkohol pro Tag) von $8.1 \%$ auf $11.7 \%$, während gleichzeitig die Zahl der abstinenten Frauen zurückging (Bundesministerium für Gesundheit, 2001). Es könnte sein, dass die Konvergenzhypothese nicht für alle Frauen, sondern lediglich für berufstätige Frauen in höheren sozialen Schichten zutrifft. In Studien aus der Bundesrepublik Deutschland, aus anderen europäischen Staaten sowie den USA stellte sich heraus, dass bei Frauen (nicht aber bei Männern) mit der Bildung der Alkoholkonsum steigt: Frauen der höchsten Ausbildungsschicht trinken signifikant mehr als Frauen der untersten Ausbil- 
dungsschicht (Härtel, Stieber \& Keil, 1993). Auch scheint das Risiko von starkem Trinken und Problemtrinken bei berufstätigen Frauen höher zu sein als bei Hausfrauen (Waldron, 1988). Diese Befunde deuten darauf hin, dass eine gleichberechtigtere Teilnahme von Frauen am gesellschaftlichen und politischen Leben mit einem Anstieg des Alkoholkonsums bei Frauen einhergeht.

Starkes Trinken, Trinken in der Öffentlichkeit sowie Betrunkensein sind bei Frauen nach wie vor ungleich stärker sozial geächtet als bei Männern; von daher ist $\mathrm{zu}$ vermuten, dass der tatsächliche Alkoholkonsum sowie alkoholbezogene Probleme bei Frauen eher unterschätzt werden. Dies wird insbesondere dann der Fall sein, wenn entsprechende Prävalenzraten auf Selbstangaben beruhen (wie z. B. die oben zitierten durchschnittlichen Trinkmengen von Frauen und Männern, die im Bundes-Gesundheitssurvey von 1998 ermittelt wurden). So ist eine alternative Interpretation des Konvergenzphänomens, dass dieses weniger auf eine Zunahme des Trinkens, sondern eher auf eine Abnahme des „Underreporting" bei Frauen zurückzuführen ist (Bloomfield et al., 2001). Frauen, die von ihrem Geschlechtsrollen-Selbstkonzept und ihrem gesellschaftlichen Status her traditionelle Geschlechtsrollenerwartungen überwunden haben, können möglicherweise offener über ihren tatsächlichen Alkoholkonsum sprechen als Frauen, die traditionellen Geschlechtsrollenerwartungen zu entsprechen versuchen.

Bei den präventiven Verhaltensweisen findet sich ein komplementäres Bild zu den Risikoverhaltensweisen: Frauen verhalten sich im Allgemeinen gesundheitsbewusster als Männer, und sie nutzen deutlich mehr professionelle Angebote zur Gesundheitsförderung und Krankheitsprävention. Im Folgenden wird kurz auf Geschlechtsunterschiede in der Inanspruchnahme professioneller Hilfe eingegangen, welche noch deutlich ausgeprägter sind als die in gesundheitsbewusstem Verhalten. Gut dokumentiert ist ein Frauenüberschuss bei Arztbesuchen (Robert-Koch-Institut, 2000), der nicht allein auf reproduktive Faktoren zurückzuführen ist. Frauen sind offensichtlich bei leichteren oder psychosomatischen Symptomen eher bereit, einen Arzt oder eine Ärztin zu konsultieren. Besonders große Unterschiede zeigen sich bei Maßnahmen der sekundären Prävention. So werden im "Gesundheitsbericht für Deutschland“ große Geschlechtsdifferenzen in der Teilnahme an Krebsfrüherkennungsuntersuchungen berichtet. Zwischen 1985 und 1995 sind die Teilnahmequoten der Frauen stetig gestiegen, die der Männer haben sich nur geringfügig erhöht. Danach nahmen 1995 nur 14\% der anspruchsberechtigten Männer an einer Krebsfrüherkennungsuntersuchung teil, dagegen fast die Hälfte (48\%) der anspruchsberechtigten Frauen (Statistisches Bundesamt, 1998).

Männer zieren sich auch deutlich mehr als Frauen, die seit den 80er Jahren verstärkt auf den Markt gekommenen Angebote zur Gesundheitsberatung und 
-förderung (z. B. Nichtraucherkurse, Ernährungs- oder Bewegungskurse oder Kurse zur Stressbewältigung) in Anspruch zu nehmen. Dass die Motivation zur Inanspruchnahme professioneller Hilfe zur Gesundheitsförderung mit Geschlechtsrollenvariablen zusammenhängt, konnte in einer Studie mit Medizinstudierenden und Klinikärzten und -ärztinnen demonstriert werden (Sieverding, 1997). Unabhängig von der Höhe der objektiven wie subjektiven beruflichen Belastung waren die Mediziner deutlich weniger motiviert, an einem Training zur Stressbewältigung teilzunehmen als die Medizinerinnen. Dabei spielte die Ähnlichkeit des Selbstkonzeptes zum Marlboro-Mann-Prototypen eine entscheidende Rolle zur Vorhersage der männlichen (nicht aber der weiblichen) Teilnahmemotivation. Männer, die in ihrem Selbstkonzept dem Marlboro-Mann aus der Kinowerbung ähnelten, konnten sich deutlich weniger vorstellen, einen Stressbewältigungskurs zu besuchen im Vergleich zu Männern, die dem Marlboro-Mann eher unähnlich waren. Eine typische Antwort zur Erklärung war: „Ich bewältige meinen Stress selbst!“ Weiterhin stellte sich heraus, dass das Image dieser Art präventiven Verhaltens nicht sehr positiv ist. So waren die freien Assoziationen zum "typischen Teilnehmer eines Stressbewältigungskurses" mehrheitlich negativ und umschrieben das Bild eines ängstlichen Versagers: das genaue Gegenbild zum männlichen Idealbild.

\section{Ausblick}

Das Anliegen dieses Beitrages war es, das Potenzial psychologischer Forschung für die Erklärung von Geschlechtsunterschieden in Gesundheit und Krankheit aufzuzeigen. Dabei wurde davon ausgegangen, dass gesellschaftliche GenderKonstruktionen nicht direkt auf Männer und Frauen „einwirken“, sondern über psychologische Variablen vermittelt werden. Geschlechtsrollenabhängige Persönlichkeits- und Selbstkonzeptmerkmale sowie die Einstellungen und das Verhalten von Interaktionspartnern vermögen das gesundheitsrelevante Verhalten eines Individuums zu beeinflussen. Einige Studien haben solche Zusammenhänge bereits überzeugend nachweisen können (Conen \& Kuster, 1988; Kohlmann et al., 1997; Sieverding, 1997, 2002; Vögele et al., 1997). Das hier vorgestellte Modell kann als Anregung für weitere dringend notwendige Forschung dienen. Forschungsbedarf besteht beispielsweise zum Zusammenhang zwischen geschlechtstypischen Persönlichkeits- und Selbstkonzeptvariablen einerseits und (physiologischer) Stressreaktivität und Krankheitsverhalten andererseits. Auch die Bedeutung sozialpsychologischer Prozesse für Geschlechtsunterschiede in gesundheitsrelevantem Verhalten sollte differenzierter untersucht werden. Hier könnten experimentelle Studien von großem Nutzen sein.

Die Berücksichtigung psychologischer Variablen ist weiterhin unter präventiven Aspekten unerlässlich. Die hohe Quote vermeidbarer Todesfälle bei Männern 
im mittleren Lebensalter stellt eine besondere Herausforderung für die Gesundheitspsychologie dar. Die Kardinalfrage lautet: Wie können Männer im jüngeren und mittleren Lebensalter motiviert werden, sich weniger gesundheitsriskant zu verhalten und mehr auf ihren Körper und ihre Gesundheit zu achten? Die enge Koppelung von Männlichkeit mit gesundheitlichem Risikoverhalten (Rauchen, Trinken, Reckless Driving) und das zum Teil negative Image von präventivem Verhalten (Sieverding, 1997) stellen hier ernstzunehmende Barrieren für die Konzeptionalisierung von effektiveren Maßnahmen zur Gesundheitsförderung und Prävention bei Männern dar. Ein weiteres wichtiges Thema (nicht nur) für die Forschung ist die weitere Entwicklung des gesundheitsrelevanten Verhaltens bei Frauen. Hier ist zu befürchten, dass bisherige Gesundheitsvorteile von Frauen abnehmen, je mehr sie sich in ihrem beruflichen Verhalten und in ihren (idealen) Selbstkonzepten dem typisch männlichen Muster annähern.

\section{Literatur}

Bloomfield, K., Gmel, G., Neve, R. \& Mustonen, H. (2001). Investigating gender convergence in alcohol consumption in Finland, Germany, The Netherlands, and Switzerland: A repeated survey analysis. Substance Abuse, 22, 39-53.

Brähler, E., Schumacher, J. \& Felder, H. (1999). Die Geschlechtsabhängigkeit von Körperbeschwerden im Wandel der Zeit. In E. Brähler \& H. Felder (Hrsg.), Weiblichkeit, Männlichkeit und Gesundheit (2. Aufl., S. 171-185). Opladen: Westdeutscher Verlag.

Bundesministerium für Gesundheit. (2001). Sucht-und Drogenbericht 2000. Unveröff. Bericht, Berlin.

Conen, D. \& Kuster, M. (1988). Geschlechts- oder symptomspezifisches Verhalten männlicher Assistenzärzte? Sozial- und Präventivmedizin, 33, 167-172.

Courtenay, W. H. (2000). Constructions of masculinity and their influence on men's well-being: A theory of gender and health. Social Science \& Medicine, 50, 1385-1401.

Deaux, K. \& LaFrance, M. (1998). Gender. In D. T. Gilbert, S. T. Fiske \& G. Lindzey (Eds.), The handbook of social psychology, Vol. I (4th ed., pp. 788-827). New York: Random House.

Gijsbers van Wijk, C.M.T. \& Kolk, A. M. (1997). Sex differences in physical symptoms: The contribution of symptom perception theory. Social Science \& Medicine, 45, 231-246.

Härtel, U., Stieber, J. \& Keil, U. (1993). Der Einfluss von Ausbildung und beruflicher Position auf Veränderungen im Zigarettenrauchen und Alkoholkonsum: Ergebnisse der MONICA Augsburg Kohortenstudie. Sozial-und Präventivmedizin, 38, 133-141.

Kohlmann, C.-W. (1997). Persönlichkeit und Emotionsregulation. Bern: Huber.

Kohlmann, C.-W., Weidner, G., Dotzauer, E. \& Burns, L. R. (1997). Gender differences in health behaviors: The role of avoidant coping. European Review of Applied Psychology, 47, $115-120$.

Lemle, R. \& Mishkind, M. E. (1989). Alcohol and masculinity. Journal of Substance Abuse Treatment, 6, 213-222. 
Macintyre, S., Hunt, K. \& Sweeting, H. (1996). Gender differences in health. Are things really as simple as they seem? Social Science \& Medicine, 42, 617-624.

Pennebaker, J. W. (1982). The psychology of physical symptoms. New York: Springer.

Robert-Koch-Institut. (2000). Public Use File BGS98, Bundes-Gesundheitssurvey 1998. Berlin: Robert-Koch-Institut.

Safran, D. G., Rogers, W. H., Tarlov, A. R., McHorney, C. A. \& Ware, J.E. (1997). Gender differences in medical treatment: The case of physician-prescribed activity restrictions. Social Science \& Medicine, 45, 711-722.

Seymour-Smith, S., Wetherell, M. \& Phoenix, A. (2002). „My wife ordered me to come“: A discursive analysis of doctors' and nurses' accounts of men's use of general practitioners. Journal of Health Psychology, 7, 253-268.

Sieverding, M. (1997). Die Bedeutung von Prototype-Matching für präventives Verhalten: Ist die Teilnahme an Streßbewältigungskursen „unmännlich“? Zeitschrift für Gesundheitspsychologie, 5, 272-289.

Sieverding, M. (2002). Gender and health-related attitudes: The role of a "macho" self-concept. In G. Weidner, S. M. Kopp \& M. Kristenson (Eds.), Heart disease: Environment, stress, and gender. NATO Science Series, Series I: Life and behavioural sciences, Vol. 327 (pp. 237-250). Amsterdam: IOS Press.

Statistisches Bundesamt. (1998). Gesundheitsbericht für Deutschland. Stuttgart: Metzler-Poeschel.

Statistisches Bundesamt. (2002). Statistisches Jahrbuch für die Bundesrepublik Deutschland. Stuttgart: Metzler-Poeschel.

Vögele, C., Jarvis, A. \& Cheeseman, K. (1997). Anger suppression, reactivity, and hypertension risk: Gender makes a difference. Annals of Behavioral Medicine, 19, 61-69.

Waldron, I. (1983). Sex differences in human mortality: The role of genetic factors. Social Science \& Medicine, 17, 321-333.

Waldron, I. (1988). Gender and health-related behavior. In D. S. Gochman (Ed.), Health behavior (pp. 193-208). New York: Plenum.

Waldron, I. (1997). Changing gender roles and gender differences in health behavior. In D.S. Gochman (Ed.), Handbook of health behavior research I: Personal and social determinants (pp. 303-328). New York: Plenum.

Weidner, G. \& Collins, R. L. (1993). Gender, coping, and health. In H.W. Krohne (Ed.), Attention and avoidance (pp. 241-265). Seattle: Hogrefe \& Huber.

Weidner, G., Kopp, S. M. \& Kristenson, M. (Eds.). (2002). Heart disease: Environment, stress, and gender. NATO Science Series, Series I: Life and behavioural sciences, Vol. 327. Amsterdam: IOS Press. 